\title{
LES TRANSPOSITIONS DE LARVES DE DIFFÉRENTES CASTES D'APIS MELLIFICA DANS LES CELLULES D'AUTRES CASTES. ÉTUDE DES TYPES INTERMÉDIAIRES ENTRE REINE ET OUVRIÈRES.
}

\author{
PAR
}

\section{G. NAULLEA U}

Station de Recherches Apicoles, Bures-sur-Yvette.

En I958, VoloseVICH a publié une note assez curieuse sur le transfert de larves d'ouvrières dans des cellules de mâles dont la larve a été enlevée au préalable, mais en laissant la nourriture qui se trouve an fond de la cellule : sur 290 larves d'ouvrières transplantées, I32 cellules operculées ont donné 97 ouvrières et 3 I mâles ; dans une autre expérience, sur I6I larves d'ouvrjères, l'auteur obtient Io6 ouvrières et Io mâlıs. I, opération inverse (I54 mâles greffés dans des cellules d'ouvrières) donne 44 mâles et 6 ouvrières. I,e changement de régime correspondant au type de cellule pourrait donc changer le sexe, que les abeilles ne sauraient distinguer? Résultat qui nécessiterait évidemment d'innombrables confirmations avant de pouvoir être admis.

HAYDAK (I958) pense, au contraire, sur la base d'analyses de la nourriture, que dans les colonies pourvues d'une reine les nourrices reconnaissent le sexe des larves; car si l'on transporte des larves d'ouvrières dans des cellules de mâles, la nourriture qui leur est distribuée se rapproche davantage de celle des ouvrières que de celle des mâles. Notons toutefois qu'il est assez banal de trouver dans les colonies depuis longtemps orphelines des nymphes de mâles dans les cellules royales. VUILIAUME (I957) a d'ailleurs parfaitement montré qu'il était possible de greffer et de faire accepter des larves de mâles dans des cupules artificielles destinées à l'élevage des reines; un certain nombre sont acceptées et élevées. Malheureusement, on n'a pas procédé jusqu'à présent à un examen systématique des modifications morphologiques induites par le changement de régime, si toutefois il existe.

WEAVER (I957) transporte dans des cellules d'ouvrières des larves Annales des Abeilles. - ig6o. 
qui ont fait un certain stage dans les cellules royales. Seules les larves qui n'y ont passé qu'un jour peuvent donner des ouvrières typiques. Si on les a portées dans les cellules royales à 1'âge de $2-3$ jours et qu'on les y laisse un jour, les adultes tendront à prendre certains caractères royaux. Il en est de même si les jeunes larves royales n'ont été transférées que le second jour dans des cellules d'ouvrières. Après tous ces transferts un grand nombre de larves meurent et ne sont pas acceptées par les nourrices. Inversement, la "greffe" de larves d'ouvrières de plus en plus âgées dans les cellules royales, ne fournit des reines normales que si les jeunes larves ne dépassaient pas, au moment de la greffe, l'âge de deux jours. Avec des larves de trois jours certains individus donnent encore des reines typiques, mais beaucoup sont des intermédiaires qui ressemblent plutôt à des ouvrières.

J'ai essayé de reprendre ces travaux, spécialement dans le but de vérifier les assertions assez étonnantes de Volosevich. Mais comme le signalent divers auteturs, les transpositions de larves s'accompagnent de très grosses pertes dues sans doute pour une part à la mort de certaines larves blessées an cours des manipulations, mais surtout à ce que les ouvrières refusent souvent d'accepter les larves transplantées et en éliminent la plupart. J'ai dû étudier d'abord les facteurs de ce refus et les moyens d'y remédier.

\section{EXPÉRIENCES FAITES DANS LA RUCHE}

\section{A. - Transpositions de larves royales dans des cellules d'ouvriòres de diliérentes longueurs, en présence de reine.}

\section{I ${ }^{\circ}$ Dans des cellules longues (11 à $12 \mathrm{~mm}$.) :}

Avec des larves de différentes grosseurs, $\mathrm{j}$ 'ai fait varier le nombre de larves royales transposées de I à I5. Dans tous les cas sauf un, les résultats sont les mêmes : dès le lendemain de la transposition des larves, quels que soient leur nombre et leur grosseur, elles sont retirées des cellules par les Abeilles et on n'en retrouve plus aucune trace.

Dans un seul cas à partir de I5 larves royales très petites les Abeilles de la ruche ont conservé d'abord 8 larves qu'elles ont operculées comme des larves d'ouvrières, puis, elles n'ont laissé que 2 larves que $\mathrm{j}$ 'ai retirées après Ir jours de vie larvaire pour les mettre à éclore en cagette à l'étuve à $30-35^{\circ}$. Les 2 Abeilles sont nées au bout de 23 jours de vie larvaire et nymphale. Elles avaient l'apparence d'ouvrières mais je ne les ai pas examinées de près ; d'ailleurs la durée du développement correspond à celui d'une ouvrière. Aussi, il y a tout lieu de penser que nous avions là deux ouvrières; nous préciserons d'ailleurs cela plus loin. 


\section{$2^{0}$ Dans des cellules courtes $(6$ à $y \mathbf{m m}$.)}

J'ai également réalisé les mêmes expériences que précédemment en faisant varier et la grosseur des larves et leur nombre. Ià, dans tous les cas, les résultats obtenus sont analogues : dès le lendemain de la transposition, les larves sont retirées par les Abeilles qui ne laissent subsister aucune larve quels que soient leur grosseur et le nombre des transpositions.

On voit ici que la longueur des cellules semble jouer un rôle mais très faible, dan.s l'acceptation par les ouvrières des larves transposées.

\section{B. - En présence at en absence de la reine.}

J'ai d'abord pensé que la reine pouvait intervenir dans cette non acceptation des larves royales par les ouvrières. J'ai donc répété les mêmes expériences à la même époque dans une même ruche, en présence ou en l'absence de la Reine. La Reine pouvait être bloquée à une extrémité de la ruche au moyen d'une grille à reine.

Le même jour, j'ai greffé des lots de 3 et 5 larves royales de différentes grosseurs dans des cellules longues d'ouvrières de deux cadres. J'ai placé ces deux cadres : l'un dans la partie de la ruche qui contenait la Reine, l'autre dans la moitié dépourvue de reine. Dès le lendemain, toutes les larves transposées des deux cadres avaient disparu.

On peut en conclure que la Reine n'a aucune action sur les ouvrières en ce qui concerne l'acceptation ou la non-acceptation des larves transposées, tout au moins quand le nombre de larves introduites est inférieur à 5 (roir plus loin).

\section{$I^{0}$ Transposition de larves d'ouvrières dans des cellules d'ouvrières ne contenant pas des larves analogues.}

a) 3 larves déjà operculées transposées dans des cellules contenant des grosses larves non operculées, enlevées au préalable (fig. 2, Pl. I).

b) Même expérience avec deux larves operculées mises dans des cellules contenant des petites larves enlevées au préalable (fig. 3, pl. I).

c) 2 petites larves transposées dans des cellules contenant des larves operculées enlevées au préalable (fig. 4, pl. I).

d) 2 petites larves transposées dans des cellules qui contenaient des grosses larves non operculées (fig. 5. pl. I).

e) Larves d'ouvrières operculées transposées dans des cellules d'outvrières contenant des œufs enlevés au préalable. 
f) 3 grosses larves transposées dans des cellules qui contenaient des petites larves (fig. 6, p1. r).

g) Larves d'ouvrières petites et grosses non operculées transposées dans des cellules d'ouvrières vides.

Dans tous ces cas, les larves ont disparu dès le lendemain de la transposition sauf dans le dernier cas où les ouvrières ont conservé 6 des petites larves qu'elles ont operculées. Alors le nombre plus élevé de transpositions pourrait peut-être jouer un rôle, en même temps d'ailleurs que la mise en place dans des cellules vides. Dans les autres cas, elles contenaient toujours soit des œufs, soit des larves retirées juste avant d'effectuer les transpositions.

\section{La raison des échecs des transpositions.}

Dans ces conditions, la non-acceptation des larves transposées étant la règle, j'ai essayé d'en chercher la raison. J'ai pensé que peut-être les larves ainsi transposées ne trouvaient pas les mêmes conditions de nutrition dans les nouvelles cellules et que les onvrières s'en apercevant les faisaient disparaitre de ces cellules.

C'est alors que j'ai transposé des larves dans des cellules d'ouvrières contenant des larves analogues enlevées au préalable.

\section{$2^{0}$ Transposition dans des cellules contenant des larves analogues.}

Ici encore, j'ai essayé d'envisager toutes les combinaisons possibles :

a) 4 petites larves transposées dans des cellules contenant des petites larves enlevées au préalable (fig. $7, \mathrm{pl}$. I).

b) 3 grosses larves operculées transposées dans des cellules qui contenaient des grosses larves operculées (fig. 8, p1. I).

c) 2 grosses larves non operculées transposées dans des cellules contenant des grosses larves non operculées (fig. 9, p1. r).

Dans ces conditions, toutes les larves transposées sont rejetées dès le, lendemain. Le degré d'analogie des laries transposées avec les larves habitant d'abord les cellules ne semble donc pas avoir d'action sur l'acceptation.

Transposition des larves avec des outils familiarisés 24 heures dans la ruche.

I e phénomène de "familiarisation " et son importance dans la vie de la ruche ont déjà attiré l'attention de V'illaume (I958).

Les outils utilisés pour le transfert des larves pouvaient être souillés de substances répulsives ou irritantes par les ouvrières, qui retiraient alors les larves touchées par ces outils. J'ai donc laissé mes outils à "familiariser " 24 heures dans la ruche et j'ai réalisé les mêmes expériences que précédemment. Dans tous les cas, les résultats obtenus sont 
identiques et la non-acceptation des larves transposées est la règle. Y.es outils "familiarisés " ne favorisent donc pas l'acceptation des larves transposées.

J'ai alors pensé que la position des larves dans le fond des cellules pouvait jouer un rôle. En effet, dans les conditions biologiques normales toutes les larves, qu'elles soient grosses ou petites avant d'être operculées, sont toujours enroulées et bien appliquées au fond de leur cellule. Ein transposant les larves dans les cellules il est très difficile sinon impossible de les mettre exactement dans la position habituelle sans les léser.

\section{Larves simplement déplacées dans leur cellule.}

Alors que normalement les larves gisent enroulées à plat au fond des cellules, je les ai placées perpendiculairement, par exemple la tête tournée vers l'ouverture des cellules. J'ai réalisé ces expériences avec des larves de différentes grosseurs.

a) 6 petites larves déplacées (fig. IO, pl. I).

b) 7 grosses larves déplacées (fig. II, p1. I).

c) 6 larves moyennes déplacées (fig. I2, pl. I).

Dans ces conditions, le pourcentage d'acceptation est beaucoup plus élevé. I a position de la larve dans la cellule joue certainement un rôle puisque certaines larves déplacées sont évacuées par les ouvrières. Cependant, ce n'est pas le seul facteur puisque un pourcentage assez important de larves déplacées est accepté.

\section{Addition de miel ou de mélange de gelée royale + eau sur les larves d'ouvrières.}

J'ai aussi essayé de déposer du miel et un mélange de gelée royale et d'eau en quantité égale sur des larves. Si leur seul contact avecles larves suffisait pour que les ouvrières les fassent disparaitre, il est possible que l'interposition d'une petite quantité de substance nutritive retarde cette réaction ou même l'inhibe complètement.

Io Sur des larves moyennes:

a) addition de miel sur 5 larves (fig. I3, pl. I).

b) addition de gelée royale + eau sur 5 larves (fig. I4, pl. I).

$2^{0}$ Sur des grosses larves:

a) addition de miel sur 4 larves (fig. $\mathrm{I}_{5}, \mathrm{pl}$. I).

b) addition de gelée royale + eau sur 3 larves (fig. I6, p1. I).

Dans tous ces cas, il y a un pourcentage d'acceptation très élevé. En général, les abeilles sucent le miel ou le mélange gelée royale + eau déposé sur les larves et laissent celles-ci à leur place. 
Transpositions de larves puis addition de miel au-dessus.

a) 6 larves transposées puis addition de miel (fig. I7, p1. I).

b) 3 larves transposées puis addition de miel (fig. I8, p1. I).

c) 5 larves transposées puis addition de miel (fig. Ig, pl. I).

d) 6 larves transposées puis addition de miel (fig. 2o, p1. I).

Dans tous les cas, la non-acceptation des larves transposées est générale et complète. Donc l'addition de miel n'augmente pas le pourcentage des acceptations après transposition.

J'ai aussi soulevé et désoperculé des larves et des nymphes avec des outils familiarisés 24 heures dans une ruche et non familiarisés.

Io Aiec outils non familiarisés :

a) 3 larves operculées désoperculées et soulevées (fig. 2I, pl. I).

b) 5 larves operculées désoperculées (fig. 22, pl. I).

c) 5 nymphes de $\sigma^{x}$ désoperculées (fig. 23, p1. 2).

$2^{\circ}$ Avec outils familiarisés :

a) 2 larves operculées et une nymphe d'ouvrières désoperculées et soulevées (fig. 24, pl. 2).

b) I nymphe d'ouvrière désoperculée (fig. 25, p1. 2).

c) 6 larves operculées désoperculées (fig. 26, pl. 2).

Ces expériences, rappelons-le, ont été faites dans des ruches normales et pourvues d'une reine. Dans tous les cas les larves désoperculées et soulevées ou non sont retirées par les ouvrières; quant aux nymphes celles qui sont désoperculées et soulevées sont retirées, celles qui sont seulement désoperculées sont réoperculées dans tous les cas. Ici encore la familiarisation des outils employés ne semble jouer aucun rôle.

Remarque: En effectuant les mêmes expériences en ruchette avec des muclei d'abeilles orphelines, celles-ci semblent réagir autrement. En effet, non seulement les nymphes operculées désoperculées sont réoperculées mais aussi les larves operculées, alors que dans la ruche normale ces larves étaient évacuées des cellules.

Deux facteurs peuvent être invoqués pour expliquer ces différences, la présence de la reine ou le nombre d'ouvrières, très réduit dans les nucléi par rapport à celui des ruches normales. Ainsi la reine, dans une ruche normale, augmenterait la sensibilité des ouvrières à toute manipulation du couvain.

Pourtant, dans tous ces transferts de larves, j'ai réussi à mettre en évidence un transport spontané de larves de la part des ouvrières.

Remarque I : Transport naturel des larves par les ouvières.

a) 2 larves transposées naturellement par les ouvrières (fig. 6, pl. I).

b) I larve transposée naturellement par les ouvrières (fig. 27, p1. 2). 
c) 6 larves transposées naturellement par les ouvrières (fig. 28, pl. 2).

d) 4 larves transposées naturellement par les ouvrières (fig. 29, pl. 2).

J'ai remarqué en faisant mes transferts de larves que certaines cellules vides le jour du transport contenaient des larves le lendemain (larves de grosseur supérieure à celle des larves de 24 heures) et certaines cellules contenant des larves la veille, sont vides le lendemain sans qu'on les ait perturbées d'une façon quelconque. Cela semble avoir l'effet d'uniformiser et d'élargir des plaques de couvain contenant des cellules vides, soit sur les bords, soit au milieu de ces plaques. On peut même remarquer que certaines cellules contenant du miel ou du pollen sont vidées, peutêtre pour y mettre des nouvelles larves (fig. I2, I3, I4, I5, p1. I).

On peut expliquer ce phénomène de la façon suivante : dans les conditions normales, lorsque la reine a à sa disposition des cadres vides, elle $\mathrm{y}$ pond au centre, formant ainsi une plaque régulière de convain. Lorsqu'il n'y a plus de place sur les cadres vides, la reine pond alors dans les cellules vides disponibles, sur les cadres, disséminées parmi des cellules contenant du miel ou du pollen. I es larves éclosent alors et les ouvrières les transporteraient ailleurs, à mesure que les éclosions se font, pour former une plaque uniforme de couvain et même, videraient certainement les cellules du pollen ou du miel qu'elles contiennent afin de régulariser au maximum la plaque de convain.

\section{Remarque II : Transport naturel des cenfs.}

J'ai aussi essayé de voir s'il n'y avait pas un transport naturel possible des cufs mais ici l'expérience est difficile et délicate; en effet des cellules vides un jour peuvent contenir des œufs le lendemain sans qu'il $\mathrm{y}$ ait eu pour cela transport d'œufs car la reine peut y venir pondre directement (fig. 3o, p1. 2).

Remarque III : Transposition d'cufs.

Pour voir si les abeilles étaient aussi sensibles au déplacement des œufs dans leurs cellules ou au transfert de ces œufs dans d'autres cellules, j'ai effectué quelques expériences.

- Eufs soulevés dans leurs cellules et remis en place:

4 œufs souvelés (fig. 30, p1. 2).

- Giufs transposés dans d'autres cellules:

7 œufs transposés (fig. 3I, pl. 2).

Dans tous ces cas, l'acceptation des œufs déplacés ou transposés est presque totale et le peu d'œufs disparus peut s'expliquer par les blessures au cours des manipulations (ils sont en effet très fragiles) et là, les ouvrières les élimineraient immédiatement.

On peut dire qu'ici les ouvrières sont insensibles à la perturbation que l'on a fait subir aux cuts, 
Remarque IV : Transfert de larves par quantités importantes.

'Toutes les expériences précédentes ayant été faites sur des larves en petit nombre, j'ai essayé d'augmenter le nombre des lavves transposées en une seule fois. Ainsi, j'ai transposé roo larves d'ouvrières de différentes grosseurs au milieu d'une plaque de couvain pour réduire au minimum le transfert naturel des larves. Le lendemain il restait 30 larves avec les proportions de Io grosses, Io moyennes et Io petites.

J'ai recommencé 1'expérience avec Ioo et le lendemain il restait 28 larves (5 grosses, 9 moyennes, I4 petites).

J'ai continué avec 64 larves, le lendemain il restait io larves moyennes et I petite.

On peut augmenter le pourcentage d'acceptation des larves transposées de la part des ouvrières en augmentant le nombre des larves transférées. En effet, alors que pour des lots de moins de I5 larves, le pourcentage d'acceptation est à peu près 1nul, pour des lots de 60 à Ioo, il varie de $I_{5}$ à 30 p. Ioo. Et 1'on peut penser que peut-être à partir d'un certain nombre limite, sans doute très élevé d'ailleurs, de larves transposées, le pourcentage approcherait de roo p. Ioo sans jamais l'atteindre (sans doute à cause du transfert spontané des larves impossible à éviter). Je n'ai pu trouver ce nombre limite, n'ayant jamais transposé plus de roo larves au maximum.

De plus, les grosses larves en petits lots sont toujours évacuées alors qu'il subsiste un pourcentage appréciable dans les lots plus importants. Et il semble bien que dans les petits lots de larves, les grosses sont d'abord éliminées, puis les larves moyennes et enfin les petites; et s'il n'en susbiste que quelques-unes, ce sont toujours des petites. A mesure que le nombre de larves transposées augmente, la possibilité d'acceptation des larves deviendrait plus grande d'abord pour les petites puis les moyennes et enfin les grosses qui, à partir de lots suffisants, pourraient subsister en nombre notable.

\section{EXPÉRIENCES FAITES SUR NUCLEUS ORPHELIN EN RUCHETTE}

J'ai fait des transferts de larves de mâles, ouvrières et reines dans différentes sortes de cellules vides en l'absence de tout autre couvain.

\section{A. - Expériences efilectuées sur un même nueleus orphelin.}

- J'ai greffé d'abord $60+63$ larves royales dans des cellules de mâles ; le lendemain il n'en reste aucune.

- Puis 98 larves d'ouvrières dans des cellules de mâles, le lendemain il reste Io larves; j'en transporte alors 54 autour de ces larves 
acceptées et le lendemain il reste 40 larves en tout. Après I 5 jours de vie larvaire, il reste 3 I larves qui sont operculées. Je les enlève alors et les mets à éclore en cagette à l'étuve à $30-35^{\circ}$ (car le but de ces expériences est l'étude comparative des individus obtenus après transposition de larves et la recherche d'intermédiaires par exemple entre reines et ouvrières).

- 60 larves d'ouvrières dans des cellules d'ouvrières au bout de 6 jours, il ne reste que 2 larves operculées.

- 80 larves de mâles dans des cellules d'ouvrières : au bout de 2 jours, il ne reste aucune larve.

- $\% 0$ larves de reines dans des cellules d'ouvrières : au bout de 3 jours, il ne reste aucune larve.

A mesure que les larves disparaissaient j'en rajoutais de nouvelles. Ainsi, j'ai remis Ioo larves de reines dans des cellules d'ouvrières et 56 larves d'ouvrières dans des cellules de mâles : le lendemain, il ne restait plus aucune larve.

De ces expériences, j'ai pu tirer quelques conclusions : tout d'abord les ouvrières ne semblent accepter définitivement qu'une seule sorte de larves transposées; dans les cas précédents, elles n'ont accepté que des larves d'ouvrières transférées dans des cellules de mâles.

D'autre part, on remarque que, pour conclure à l'acceptation définitive des larves transposées, il faut compter un délai de 2 à 3 jours, avec les ouvrières du nucléus orphelin.

Dans le cas où le lot de larves était accepté, les ouvrières étaient toujours en train d'ébaucher des cellules royales; cela semble favoriser d'ailleurs l'acceptation des larves transposées.

D’après les résultats de ces expériences, par la suite, j'ai été amené à faire une seule sorte de transposition de larves par nucléus pour augmenter le pourcentage des acceptations.

\section{B. - Une seule expérience par nucléus orphelin.}

Nucléus 1: J'ai transposé roo larves de reines dans des cellules d'ouvrières : au bout de 5 jours, il reste 8 larves dont les cellules sont operculées. Ces cellules sont alors protégées d'une petite cage de toile métallique, pour éviter qu'elles ne soient détruites par la suite par les ouvrières. Puis, après I6 jours de vie larvaire, les cellules sont mises à éclore en cagette à l'étuve à $30-35^{\circ}$.

Remarquons tout de suite que les larves sont operculées différemment :

- 2 sont operculées comme des cellules royales typiques,

- I est operculée comme une cellule royale mais avec un capuchon beaucoup plus court. 
Les 5 autres enfin sont operculées comme des ouvrières avec cependant des opercules plus ou moins bombés.

Nucléus 2 : Ici j'ai transposé $50+50$ larves royales dans des cellules de mâles : au bout de 3 jours il reste $3+5$ larves operculées mises en cage comme précédemment. Certaines sont operculées en reines typiques, d'autres comme des ouvrières avec opercule plus ou moins bombé.

Nucléus 3: J'ai transféré Ioo larves de mâles dans des cellules d'ouvrières : au bout de 8 jours, il reste I 3 larves operculées toutes comme des mâles, c'est-à-dire avec des opercules bombés. Elles sont aussi mises en cage pour éviter leur destruction.

\section{Remarque I : Influence de la saison.}

Au début de septembre, j’ai transposé des lots de 8 o larves, d'abord des larves royales dans des cellules d'ouvrières, des larves royales dans des cellules de mâles et enfin des larves d'ouvrières dans des cellules de mâles. Dans tous les cas, dès le lendemain de la transposition, toutes les larves avaient disparu.

Nous voyons que le pourcentage d'acceptation des larves transposées en grand nombre est maintenant égal à zéro. Cela est probablement dî̀ à la saison car souvent, à partir de la fin d'aôt ou au début de septembre, une pénurie de nourriture commence à se manifester et les ouvrières sont obligées de détruire les larves : sans doute les larves transposées sont-elles sacrifiées les premières? J'ai aussi essayé de greffer des larves de mâles dans des ébauches de cellules royales sur mélange de gelée royale et d'eau pour essayer d'étudier la morphologie des individus que l'on obtiendrait à partir de ces larves. Vurluatine (I957) avait réalisé avec succè: ces mêmes expériences, pour comparer les formes des cellules royales dans lesquelles il avait greffé des larves de mâles. Cette année, il n'y a eu aucune possibilité d'obtenir des cellules royales avec des larves de mâles à l'intérieur: les larves ne sont jamais acceptées ou alor: se font détruire très tôt. Peut-être cet échec est-il dû lui aussi aux caractères très particuliers de la saison apicole 1959?

Remarque II : Action du gaz carbonique sur les larves.

J'ai aussi essayé de voir si l'action du gaz carbonique sur les larves était suffisante pour qu'elles soient détruites par les ouvrières. Pour cela, dans un rayon de couvain bien uniforme contenant des larves de toutes les grosseurs, operculées ou non, et même des oufs, j’ai découpé un fragment de rayon qui possédait d'un côté 60 grosses larves d'ouvrières et I4 larves operculées, de l'autre, 6o larves de petite taille et de taille moyenne. Ce rayon a été mis en atmosphère de gaz carbonique pur pendant 5 minutes puis remis aussitôt à sa place dans le cadre et introduit dans la ruche. I,e lendemain, on remarque d'un côté 6 grosses larves 
non operculées, 3 petites et 62 larves operculées; de 1'autre côté, 4 petites larves qui devaient être la veille à l'état d'œufs.

D'après cette expérience, le gaz carbonique doit avoir une action sur les petites et moyennes larves puisqu'elles sont détruites par les ouvrières mais non sur les œufs, ni sur les grosses larves, qui sont operculées normalement.

\section{EXPÉRIENCES FAITES EN CAGETTES}

J'ai aussi élevé des larves transposées en cagettes à l'étuve à 30-35 . I es cagettes contenaient Ioo onvrières prises dans la ruche, nourries avec du miel et du pollen de cadre. Les morceaux de rayons contenant les larves transposées, ont été introduits dans les cagettes après un séjour des ouvrières de 3 à 4 heures dans la cage. Nous avions réalisé les mêmes expériences en I958 avec VUILLA 'Mie pour étudier la transformation de la cellule en fonction de la larve transposée. Cette année, j'ai transféré des lots d'une cinquantaine de larves dans les mêmes conditions : larves de reines dans des cellules de mâles, des larves d'ouvrières dans des cellules de mâles et des larves de reines dans des cellules d'ouvrières. Dans tous les cas, il n'y a en aucune acceptation de larves transférées.

En conclusion, nous pouvons essayer de dégager les conditions, plus ou moins favorables, intervenant dans l'acceptation des larves. Tout d'abord un facteur essentiel est la longueur des cellules : les transpositions doivent toujours se faire dans des cellules longues de mâles ou d'ouvrières (cellules dont la construction est achevée). Il est d'ailleurs rare sinon impossible de trouver des larves dans des cellules courtes dans les conditions normales. Le nombre semble le plus important : en effet pour les petits lots, la non acceptation est toujours la règle alors que si l'on augmente le nombre de larves transposées par lot, le pourcentage d'acceptation croit. C'est ainsi que pour des lots de Ioo larves on peut obtenir 30 p. roo d'acceptation. D'autre part, dans des lots peu importants, ce sont d'abord les petites larves qui sont acceptées. De plus, le seul contact mécanique des outils avec les larves semble diminuer l'acceptation des larves transposées alors que l'action d'un liquide (miel ou mélange gelée royale + eau) ne gêne absolument pas 1'acceptation. L'action du gaz carbonique suffit à faire disparaitre les larves mais les petites seulement; son action doit d'ailleurs se limiter aux petites larves qui sont tuées et alors évacuées par les ouvrières. D'autre part, la présence de la reine semble inhiber complètement l'acceptation des larves transposées en petits lots, puisqu'elles ne sont acceptées dans aucun cas. Pour des lots assez importants, la reine diminue le pourcentage d'acceptation des larves transposées. En effet, avec des trans- 
positions effectuées dans des nucléi orphelins on obtient des pourcentages d'acceptation plus élevés, en particulier lorsque le transfert ne porte que sur une catégorie de larves.

De toute façon, dans les meilleures conditions, le pourcentage d'acceptation des larves transposées pour des lots d'une centaine est toujours inférieur à 50 p. IOo. A quoi ce fait peut-il être dû? Le nombre joute certainement un grand rôle (d'ailleurs difficile à comprendre) mais ce n'est pas le seul facteur. La position de la larve dans la cellule est importante aussi et 1'on ne peut sans doute rétablir exactement la position normale au cours des transpositions. De plus il peut arriver qu'un certain pourcentage, vraisemblablement assez faible, soit blessé par les manipulations. Peut-être faut-il songer aussi à une excitation nerveuse anormale qui se déclenche chez la larve transposée après contact mécanique, trouble ses réactions quand la nourrice s'approche et provoque l'expulsion, mais on ne comprend guère l'intervention d'un tel mécanisme nerveux sur les nymphes immobiles; et pourtant une nymphe simplement désoperculée est réoperculée normalement. Cependant, des oufs transposés sont évidemment parfaitement immobiles et ne sont pas éliminés par les ouvrières. Une interaction des différents facteurs énumérés plus haut et non pas un facteur isolé doit pouvoir rendre compte des expulsions des larves; mais celle des nymphes est beaucoup plus difficile à expliquer.

\section{LES MODIFICATIONS ANATOMIQUES DES ADULTES PROVENANT DE LARVES TRANSPOSÉES}

Après ces multiples transpositions de larves, j'ai réussi à obtenir des nymphes et des adultes qui m'ont permis l'analyse des caractères morphologiques.

\section{Transposition de larves d'ouvrières dans des cellules de mâles.}

Planches 3 , et 4 .

Rappelons ici que les expériences ont été faites sur des muclei orphelins afin d'augmenter le pourcentage d'acceptation.

J'ai obtenu de nombreux individus adultes et j'ai pu étudier assez bien leurs caractères morphologiques (29 adultes sur I5O transpositions). Remarquons tout de suite que les cellules n'étaient pas operculées de la même façon ; en effet il y en avait d'operculées en reines typiques, d'autres en reines avec un capuchon plus ou moins long, mais de longueur toujours inférieure à celle de la reine normale, et enfin, d'autres étaient operculées en ouvrières avec un opercule plus ou moins bombé : les éclosions se sont échelonnées sur Ig jours : les adultes des cellules aux capuchons plus longs sont éclos les premiers puis ceux aux capu- 
chons les plus courts et enfin ceux des cellules à opercules plus ou moins bombés. A première vue, les premiers adultes éclos sont des reines typiques, tandis que les derniers sont des ouvrières typiques. Quant aux intermédiaires, leurs caractères morphologiques sont plus complexes.

J'ai étudié tous ces caractères sur les lots d'individus qui naissaient chaque jour :

- Morphologie de la patte postérieure et de ses griffes.

- Morphologie de la tête (longueur de la trompe, forme des mandibules et emplacement des ocelles).

- Morphologie du 6 e sternite abdominal.

- Morphologie de l'aiguillon.

I $^{0}$ Morphologie de la patte postérieure (P1. 3 et 4 ).

Les premières abeilles nées $\left(A_{1}, A_{2}, A_{3}\right)$ ont les caractères typiques de la Reine $\mathrm{R}$, et les caractères d'ouvrières n'apparaissent nettement que chez les abeilles nées à partir du $5^{\mathrm{e}}$ jour $(\mathrm{E}, \mathrm{I}, \mathrm{G} . .$.$) .$

Toutefois chez les abeilles nées dans les + premiers jours, de $A_{1}$ à $D$, on peut retrouver dans certaines pattes des caractères aberrants. Ainsi, une abeille $B_{3}$, née le $2^{\mathrm{e}}$ jour, présente dans son tibia un certain crochet (Cr.) qui n'existe d'ailleurs pas dans la patte de l'ouvrière $O$. En $B_{4}$, abeille née aussi le $2^{\mathrm{e}}$ jour, on remarque l'apparition d'un début de pince à Pollen (Pi.), caractère d'ouvrière que l'on ne retrouve d'ailleurs plus avant les abeilles $d u 5^{\mathrm{e}}$ jour $\mathrm{E}$. En $\mathrm{C}_{1}$, abeille née le $3^{\mathrm{e}}$ jour, on trouve là une patte postérieure absolument anormale, tordue, mais gardant cependant les caractères de Reine (R.), à part le $2^{\mathrm{e}}$ article du tarse $\mathrm{T}_{2}$ qui lui, est assez large et massif, ressemblant ainsi à celui de l'ouvrière O. Puis, les abeilles nées jusqu'au $4^{e}$ jour (D) ont les caractères de Reine $R$.

Ce n'est que dans les abeilles nées au $5^{\mathrm{e}}$ jour (E) que l'on aperçoit nettement les caractères d'ouvrières $(O)$ : apparition de la corbeille (Co), du peigne à pollen (Pei) et de la pince poussoir à pollen (Pi). Cependant, les articles du tarse, en particulier le $2^{\mathrm{e}}\left(\mathrm{T} . A_{2}\right)$, sont un peu différents de ceux de l'ouvrière $O$ et ressemblent beaucoup à ceux de la reine $\mathrm{R}$. Ce n'est que chez les abeilles naissant à partir du $8 \mathrm{e}$ jour $(\mathrm{H}, \mathrm{I})$ qu'apparaît vraiment la forme des tarses d'ouvrières ainsi qu'une pince poussoir à pollen typique.

\section{$2^{0}$ Morphologie des griffes des pattes postérieures (Planche 4).}

Les résultats semblent assez variables. Cependant, on peut dire que chez les abeilles nées les 6 premiers jours, de $A_{1}$ à $F$, nous retrouvons des griffes analogues à celles de Reine $\mathrm{R}$ en particulier en ce qui concerne 1'échancrure inférieure $(\mathrm{Ec})$. Et les abeilles qui naissent les trois derniers jours $G, H$. I ont des griffes analogues à celles de l'ouvrière $O$, surtout 
par l'échancrure inférieure Ec. I à aussi, dans ces 2 parties bien tranchées, il doit $\mathrm{y}$ avoir certains caractères plus ou moins aberrants qui. sont ici difficiles à mettre en évidence.

\section{$3^{0}$ Morphologie de la tête : (Planche 4). \\ Place des ocelles et forme de la tête.}

On remarque que les têtes d'abeilles nées les 4 premiers jours (de $A_{1}$ à $\left.D\right)$ sont identiques à la tête de la reine $R$. I es abeilles nées les 6 derniers jours (de E à I) ont une tête qui ressemble à celle de l'ouvrière au moins en ce qui concerne la forme de la tête et la place des ocelles.

\section{Longueur de la trompe.}

On peut remarquer une certaine évolution de la longueur de la trompe. Des abeilles, par exemple celles nées du $5^{\mathbf{e}}$ au 7 - $^{\mathbf{e}}$ jour (de Ė à G), ont des têtes apparemment d'ouvrière mais une trompe de longueur intermédiaire entre celle de la reine $R$ et celle de l'outvrière $O$ : en effet, la trompe est plus longue que celle de la reine mais plus courte que celle de l'ouvrière et ce n'est qu'à partir du $8 \mathrm{e}$ jour, (abeille H) que la trompe devient. de la longueur de celle de l'ouvrière.

\section{Morphologie des mandibules: (Planche 2).}

L es abeilles nées les 5 premiers jours (de $A_{1}$ à $E$ ) ont des mandibules analogues à celles de la Reine R. Les abeilles nées les 4 derniers jours (de $F$ à I) ont des mandibules analogues à celles de l'ouvrière $O$. 'Toutefois, là encore, la coupure n'est pas aussi absolue : ell effet, si les premières abeilles nées $\left(A_{1}\right)$ ont des mandibules typiques de Reine, les abeilles nées le $2^{\mathrm{e}}$ jour $\left(\mathrm{B}_{4}\right)$, possèdent des mandibules analogues à celles de Reine, mais déjà avec des angles qui commencent à s'arrondir ; elles

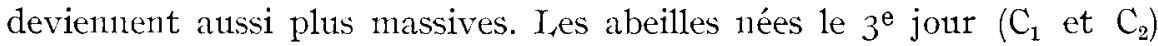
ont des mandibules de reines mais avec des angles très arrondis; elles sont massives arec l'extrémité mandibulaire (Ex.) moins longue et plus épaisse $\left(\mathrm{C}_{2}\right)$. Et, si les abeilles nées à partir du $6^{\mathrm{e}}$ jour $\left(\mathrm{F}^{\mathrm{i}}\right)$ possèdent des mandibules d'ouvrières, elles possèdent un caractère de reine : elles sont plus échancrées en $\mathrm{K}$ que les mandibules d'ouvrières et ce n'est que les abeilles nées à partir dı $7^{\mathrm{e}}$ jour $(\mathrm{G})$, qui possèdent vraiment des mandibules tỵpiques d'ouvrières.

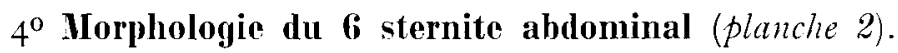

Chez les abeilles nées les 5 premiers jours (de $A_{1}$ à $E$ ), le $6^{\text {e }}$ sternite abdominal est analogue à celui de reine $\mathrm{R}$ et celles nées les 4 derniers jours (de $\mathrm{E}$ à I) ont le $6^{\mathrm{e}}$ sternite abdominal analogue à celui de l'ouvrière 
O. Mais là encore, dans ces deux parties, on retrouve certains intermédiaires. Par exemple, les abeilles nées $1 \mathrm{e} 2^{\mathrm{e}}$ jour $\left(\mathrm{B}_{\mathbf{4}}\right)$ possèdent un $6^{\mathrm{e}}$ sternite de reine mais avec apparition de caractères d'ouvrières : le bord cuticulaire supérieur $(\mathrm{Cu})$, possède un début de convexité, le bord cuticulaire latéral $\mathrm{Cu}$ est plus concave. Insertion des poils (Po, en pointillé) plus haute au centre.

Chez les abeilles nées du $3^{\mathrm{e}}$ au $5^{\mathrm{e}}$ jour (de $\mathrm{C}$ à $\mathrm{E}$ ), les caractères d'ouvrières s'accentuent (convexité du bord cuticulaire supérieur, concavité du bord cuticulaire latéral et insertion des poils). Mais, ce n'est que chez les abeilles nées à partir du $6^{\mathrm{e}}$ jour, $\mathrm{F}$ que l'on retrouve le $6^{\text {e }}$ sternum abdominal analogue à celui de l'ouvrière. Mais chez ces abeilies, on peut retrouver quelques caractères de Reine, c'est ainsi que les abeilles nées le $7^{\mathrm{e}}$ jour $(\mathrm{G})$ possèdent un bord cuticulaire latéral plus long que celui d'une ouvrière.

Et ce n'est que chez les abeilles nées le dernier jour (I) que l'on trouve un $6^{\mathrm{e}}$ sternite abdominal typique d'ouvrière.

\section{$5^{\circ}$ Morphologie de l'aiguillon,}

Ein comparant la morphologie des aiguillons de Reine $\mathrm{R}$ et d'ouvrière $\mathrm{O}$, on constate qu'ils sont totalement différents. En examinant les aiguillons des différents individus on remarque tout d'abord qu'on ne peut pas séparer et classer les individus d'après la forme de l'aiguillon et on ne peut pas dire qu'à partir de telle abeille, apparait la forme typique de 1'aiguillon d'ouvrière. On remarque une évolution progressive, des premières abeilles nées aux dernières. Les abeilles nées les 2 premiers jours $\left(\mathrm{A}_{1} \ldots \mathrm{B}_{4}\right)$ ont un aiguillon type reine tandis que celles nées le dernier jour (I) ont un aiguillon typique d'ouvrière. Entre ces deux extrêmes, il existe tous les intermédiaires. I'aiguillon de la reine $\mathrm{R}$ étant courbé vers le bas et celui de l'ouvrière vers le haut, on voit, chez les abeilles nées le $5^{\mathrm{e}}$ jour $(\mathrm{F})$ apparaître à la base de l'aiguillon de reine, la courbure vers le haut caractéristique de l'ouvrière. Puis, cette courbure semble se déplacer vers la pointe de l'aiguillon ( $F, G, H)$ pour enfin aboutir à l'aiguillon typique d'ouvrière (en $\mathrm{I}$, ).

D'après l'étıde de ces différents caractères, nous obtenons trois catégories d'individus; les premiers nés qui sont des reines typiques et les derniers nés qui sont des ouvrières typiques. Quant aux individus nés dans l'intervalle, ils sont intermédiaires. Remarquons que l'on peut distinguer aussi des variations d'autres caractères somatiques. En particulier, on voit l'abdomen se modifier dans sa forme et sa longueur. Petitêtre dans ces intermédiaires, avons-nous des "pré-reines " (reines plus ou moins régressées et même avec des caractères d'ouvrières) (DARCHEN et VuILLAUME, I959)? Il se pourrait que les organes soient eux aussi 
intermédiaires surtout l'appareil génital. Mais nous n'avons pu analyser que les caractères morphologiques externes. Nous ne savons pas non plus comment varient le comportement et la physiologie de ces intermédiaires ni s'il s'en trouve qui puissent se reproduire.

\section{Transposition de larves de reines dans des cellules d'ourrières : (Planche 4)}

Ici l'expérience a été plus difficile et le pourcentage d'individus obtenus moins grand ( 8 individus sur Ioo transplantations ; 2 éclosions seulement). Les 6 individus restants sont morts dans les cellules à l'état de nymphes ou d'adultes.

Remarquons tout de suite que les larves ont été operculées de façon différente : 2 étant en reines typiques, I en cellule royale (mais opercule plus court) et les 5 autres en ouvrières avec opercule plus ou moins bombé : les deux cellules royales contenaient des nymphes mortes. J'ai malgré tout pu comparer les caractères morphologiques des différents individus ou nymphes.

Pour les nymphes des cellules royales, je n'ai pu examiner que la tête, les nymphes étant trop jeunes pour étudier les autres caractères. La tête $\mathrm{R} \mathrm{O}$ est bien une tête typique de reine au point de vue place des ocelles, forme des mandibules et longueur de la trompe. L'aspect général de la nymphe est d'ailleurs celui d'une reine.

Quant aux cellules operculées en ouvrières, j'ai pu étudier tous les caractères morphologiques sur des individus adultes.

$I^{\circ}$ Morphologie de la tête $\left(O R_{1}\right.$ Planche 4$)$.

I a forme de la tête, la place des ocelles, la forme des mandibules et la longueur de la trompe nous font penser à une tête typique d'ouvrière.

\section{$2^{\circ}$ Morphologie de la patte postérieure et des griffes $\left(\mathrm{OR}_{3}, \mathrm{OR}_{4}\right.$, Planche 4).}

La patte est typiquement celle de l'ouvrière avec corbeille, peigne, etc... cependant le $2^{\mathrm{e}}$ article du tarse $\mathrm{T}_{2}$ a une forme aberrante un peu spéciale. Les griffes elles, semblent posséder à la fois des caractères de reine et d'ouvrière.

$3^{\circ}$ Morphologie de l'aiguillon $\left(\mathrm{OR}_{2}\right.$ Planche 4$)$.

L'aiguillon a, luii aussi, des caractères d'ouvrières et de reines avec toutefois des caractères d'ouvrières. En effet, la courbure très nette vers le haut appartient à l'ouvrière; mais à l'extrémité une courbure moins nette vers le bas ressemble à celle de l'aiguillon de reine. 


\section{$4^{\mathrm{o}}$ Morphologie du $6^{\mathrm{e}}$ sternite abdominal ( $\mathbf{O R}_{5}$ Planche 4$)$.}

Là, nous avons le $6^{\mathrm{e}}$ sternite abdominal typique de l'ouvrière sans aucun caractère de reine.

Disons tout de suite que les individus extraits des cellules operculées en ouvrières avaient l'aspect général de l'ouvrière mais toutefois, étaient de petite taille par rapport aux ouvrières normales. Nous obtenons donc 2 catégories d'individus : des reines typiques et des intermédiaires (ouvrières avec caractères de reines) ; certes les caractères d'ouvrières dominent mais nous n'obtenons pas d'ouvrières avec tous leurs caractères typiques. Cela est peut-être dûu au fait que les larves de reines transposées avaient atteint un stade de différenciation tel que tout retour en arrière pour donner des ouvrières parfaites était totalement impossible. Les individus qui possèdent le plus de caractères d'ouvrières sont sans doute issus le larves royales très petites, pas encore entièrement différenciées en larves royales.

\section{Transposition de larves de mâles dans des cellules d'ouvrières : Modifications anatomiques des nymphes. (Planche 4).}

Ici roo larves transposées n'ont donné que I4 individus. Tous sont morts dans les cellules à l'état de nymphe, à peu près au même stade. N'ayant pu obtenir d'adultes, j'ai été obligé d'étudier les caractères morphologiques des nymphes. Tout d'abord disons que les larves ont toutes été operculées avec un opercule bombé typique de mâles. J'ai pu remarquer un fait curieux en désoperculant les nymphes mortes pour les sortir; j'en ai trouvé 2 qui étaient placées la tête vers le fond de la cellule. Ce fait arrive-t-il dans les conditions naturelles? Ou est-il dù simplement à la position de la larve transplantée qui n'a pas pu se déplacer pour tourner la tête vers l'ouverture de la cellule? De toute façon, on peut remarquer que ces 2 nymphes sont mortes au même stade que les autres, par conséquent elles ont été acceptées et nourries par les ouvrières dans les mêmes conditions que les autres. Il est cependant probable qu'elles n'auraient pu éclore.

Nous avons représenté une nymphe normale avec son abdomen massif caractéristique $\left(\mathrm{M}_{2}, \mathrm{P1} .4\right)$.

La transposition ne semble pas avoir modifié les individus tout au moins dans leur morphologie externe. Nous avons en effet en $M_{1}$, P1. 4, une tête typique de mâle d'après sa forme (place des ocelles, volume énorme occupé par les yeux).

Remarque : Transposition de larves de reines dans les cellules de mâles.

Je n'ai eu ici aucune possibilité d'étudier les caractères morphologiques des individus ou des nymphes obtenus; en effet, tous les indi- 
vidus étant morts dans les cellules à l'état de larves. Sur roo larves transposées, il en est resté I3: 4 ont été operculées en cellules royales, les autres en ouvrières avec opercule plus ou moins bombé.

Il y a un fait important : c'est la grande mortalité des individus transposés à un stade d'ailleurs plus ou moins avancé. A quoi cela est-il dî̀? Peut-être à un changement de régime. Peu d'individus éclosent normalement. Nous voyons que dans 1'ensemble la transposition peut fournir des intermédiaires au point de vue de la morphologie externe.

\section{CONCLUSION ET RÉSUMÉ}

Io Les transpositions de larves sont toujours suivies d'une disparition de la moitié au moins des larves ainsi transposées.

$2^{\circ}$ On n'a quelque chance de réussite que si l'on transpose une centaine de larves au moins, le nombre des larves transposées constituant le plus important facteur de réussite.

$3^{\circ}$ Dans les nuclei pourvus d'une reine, les abeilles sont sensibles non seulement à la transposition, mais au simple déplacement d'une larve dans sa propre cellule; un certain nombre de larves ainsi déplacées (très légèrement) sont toujours éliminées. Les lésions produites par les manipulations n'en constituent pas une explication suffisante, puisqu'il suffit de désoperculer une larve operculée pour provoquer son expulsion, phénomène singulier et difficile à interpréter.

$4^{\circ}$ Dans les nuclei sans reine, la sensibilité des ouvrières aux perturbations est bien moins grande; les larves transposées sont plus souvent acceptées, et les larves désoperculées sont réoperculées, alors que dans le nucléus avec reine, seules les nymphes désoperculées étaient réoperculées.

$5^{\circ}$ On a pu mettre en évidence le transport spontané par les ouvrières des larves d'une cellule dans l'autre.

$6^{\circ}$ Les larves ont été transférées alors dans des nuclei orphelins afin d'éviter précisément ce transfert spontané. I,orsque dans ces conditions des larves d'ouvrières sont transportées dans des cellules de mâle, on n'obtient aucun mâle, mais seulement des reines et une série de formes intermédiaires assez semblables à celles décrites par GoNTARski (I94I) et Komarov (r935) et qui compliquent souvent la recherche de la reine dans une ruche. Les larves de reine transposées dans des cellules d'ouvrières donnent, elles aussi, des reines et des formes intermédiaires. Les mâles transférés dans les cellules d'ouvrières n'ont donné que des mâles, en très petit nombre. Les larves de reine transtérées dans les cellules de mâles sont toutes mortes dans les cellules après 1'operculation, mais avant la nymphose, si bien qu'on n'a pu étudier leurs caractères morphologiques ; leurs opercules correspondaient à des reines ou à des ouvrières. 
Ainsi donc, nous avons montré à quel point il est facile d'obtenir des formes intermédiaires, ce qu'avaient signalé d'ailleurs un certain nombre d'auteurs; mais surtout, nous avons pu mettre en évidence la grande sensibilité des nourrices à toute perturbation apportée aux larves, sensibilité qui paraît dépendre en grande partie de la présence de la reine.

Il est curieux de remarquer aussi que les intermédiaires sont nés à proximité des cellules royales comme si les larves voisines des reines avaient bénéficié d'un supplément de nourriture. Èn est-il ainsi dans une ruche normale et les intermédiaires ont-ils la même provenance? D'anciens auteurs l'ont prétendu.

\section{REFERENCES BIBLIOGRAPHIQUES}

Darchen (R.), Vuillaume (M.). - Déterminisme de la construction des cellules de mâles et des cellules d'ouvrières chez Apis mellifica $\mathrm{L}$. Ann. A heille, $\mathrm{n}^{\circ} \mathrm{I}, 7-9, \mathrm{r} 95^{8}$.

Darchen (R.), Vuir.iaume (M.). - Deux types de cellules " royales " chez Apis mellifica. Existe-t-il chez Apis mel. des sexues de remplacement? Ann. Abeille, 2, I77, I83, I959.

GonTARSki (H.). - Ueber Zwischenformen von Königin und Arbeiterin im Staat der Honigbiene (Apis mellifica L.). Z. Zool., 154, 345-6, I94I. Haydak (M.). - Do the nurse bees recognize the sex of the larvae? Science, $12 \%, \operatorname{III}$, I958.

Komarov (P. M.). - Uebergangsformen bei weiblichen Honigbiene. Zum Polymorphismus sozialer Insekten. Archiv Bienenk., 16, I52, 1935.

Vorosevich (A. P.). - Pschelovodstvo, p. 20-22, I955.

VUnI.AUME (M.). - Contribution à la psychophysiologie de l'élevage des reines chez les Abeilles. Ins. Soc., IV, II3-I56, I957.

- Nouvelles données sur la psychophysiologie de l'élevage des reines chez Apis mellifica. Ann. Abeille, II, I958.

WeAver (N.). - Effects of larval age on dimorphic differentiation of the female honeybee. Ann. Entom. Soc. Amer., 50, 285-94, I957. 


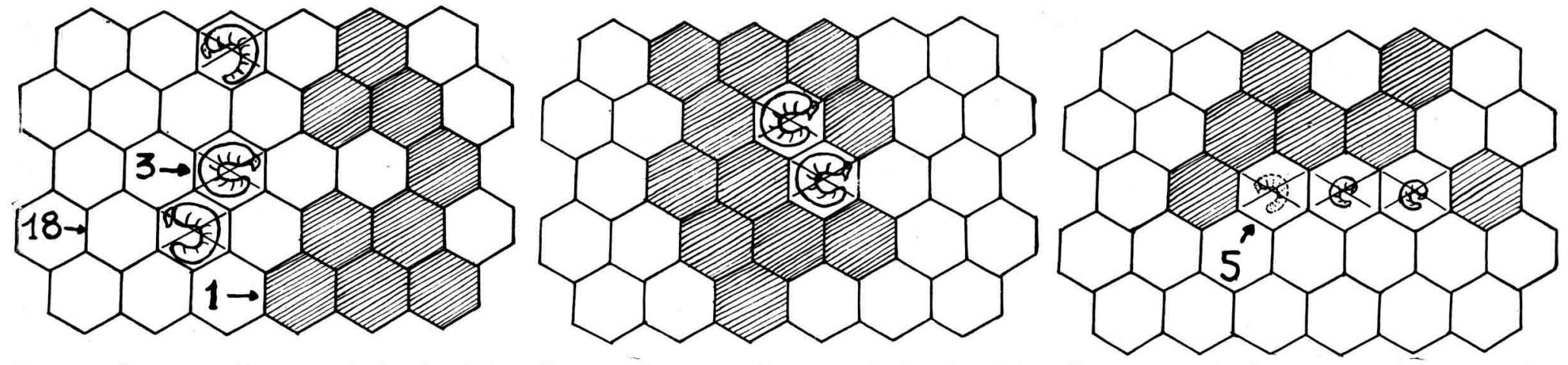

Fig. 2. - 3 larves operculées transposées dans les cellules

Fig. 3. -2 larves operculées transposées dans des cellules

Fic. $4 .-2$ petites larves non operculées transposées
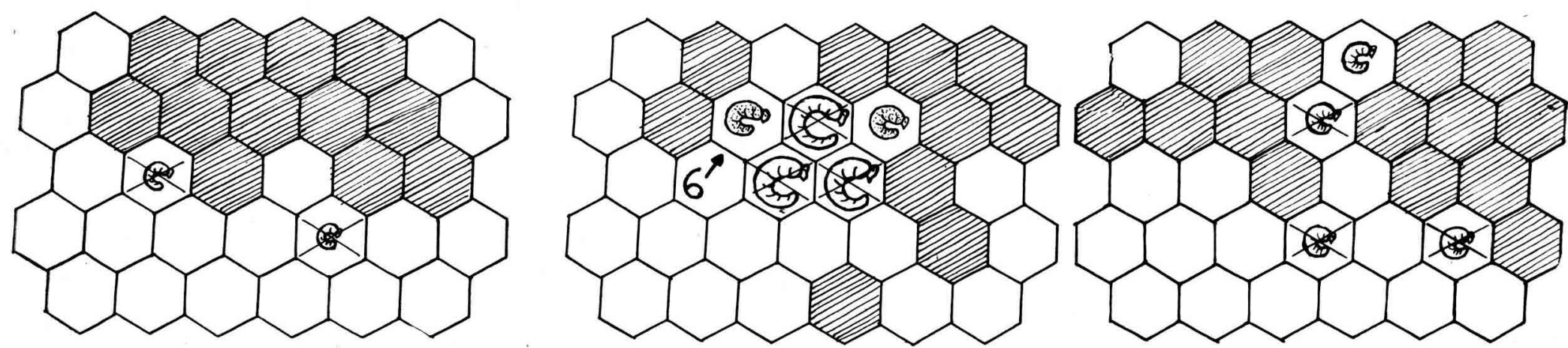

Fig. 5. - Deux petites larves transposées dans des cellules

FIG. 6. - 3 grosses larves non operculées transposées Fig. 7. -4 petites larves transposées dans des cellules dans des

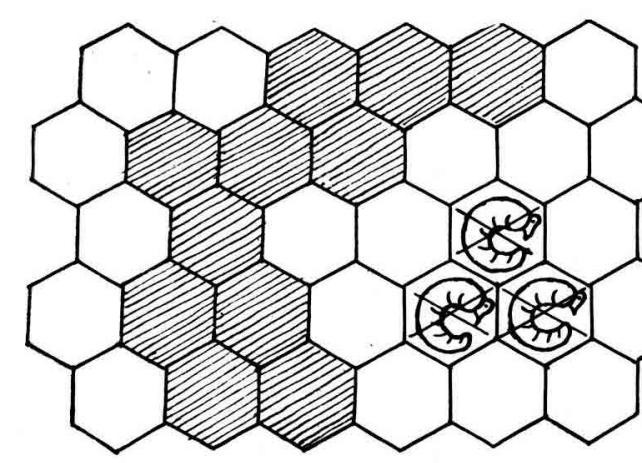

Fig. 8. - 3 larves operculées transposées dans

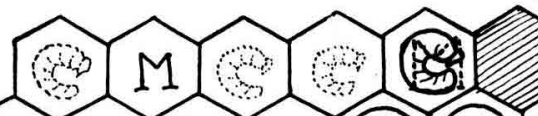

M.M.M.M M M.M.P. M M M $M$ $7 \rightarrow M M$

Fits. II. -7 grosses larves déplacees.

$\widehat{P}|\widehat{P}| \widehat{P} / P|P| \widehat{P}$ $P$ P $P$ P P i $\gg$

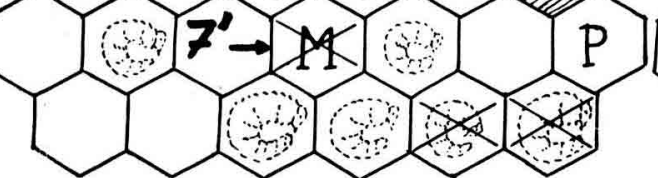

FIG. 14. -5 larves moyennes avec addition

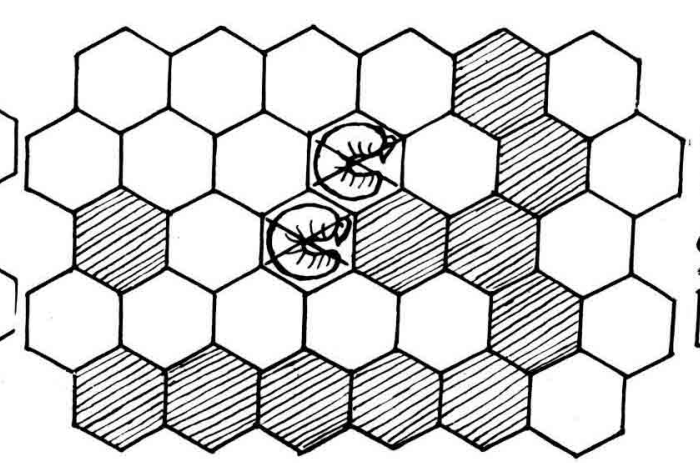

Fig. $9 \cdot-2$ grosses larves non operculées transposées
dans des cellules qui contenaient des grosses larves.

$\widehat{M} \widehat{M} \widehat{M} / \widehat{P} \widehat{P} / \mathrm{M}$ $M / M / P$ P $M$ 9. $M$ M $M$ P $\rightarrow M$ C $\because$ M M M

FIG. ro. -6 petites larves déplacées.

P/M M M M

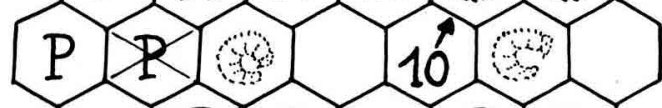

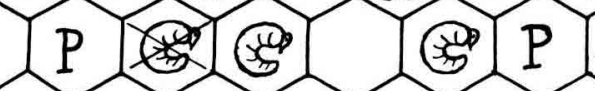

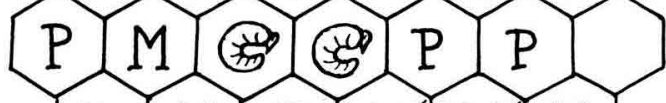
IP M P $P M$ FIG. 13. -5 larves moyennes avec addition de miel dessus. Fig. I2. - 6 larves moyennes déplacées.

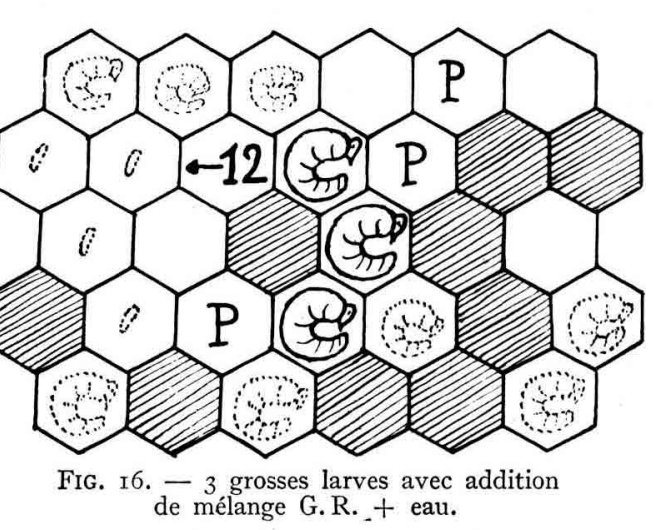

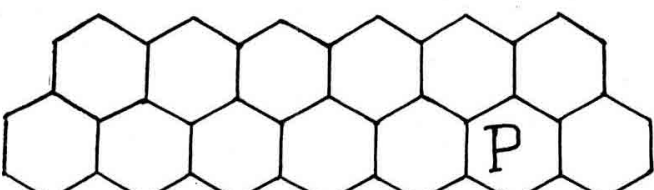
M M.MI एक $\rightarrow M$ M $14 \mathrm{M}$ m M M Fig. 15. -4 grosses larves

आ०िखिखि

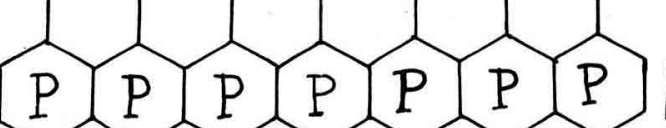
$P$ P $P$ P $P$ P P P \&PIPLPlPlP $P$ PIPIP Fig. 17. -6 larves transposées avec addition $0 P P L P I P$ FIg. 18. - 3 larves transposées avec addition
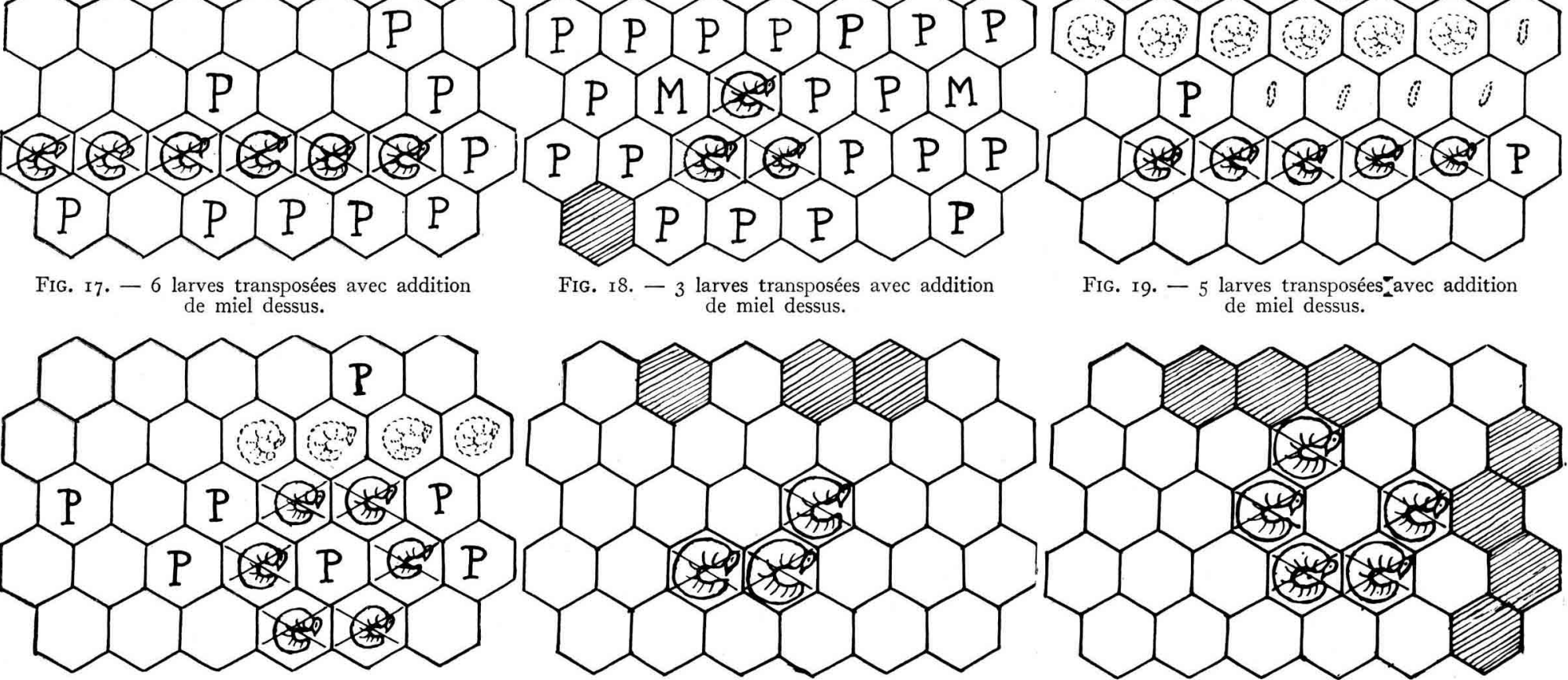

FiG. 20. - ó larves transposées avec aủủition
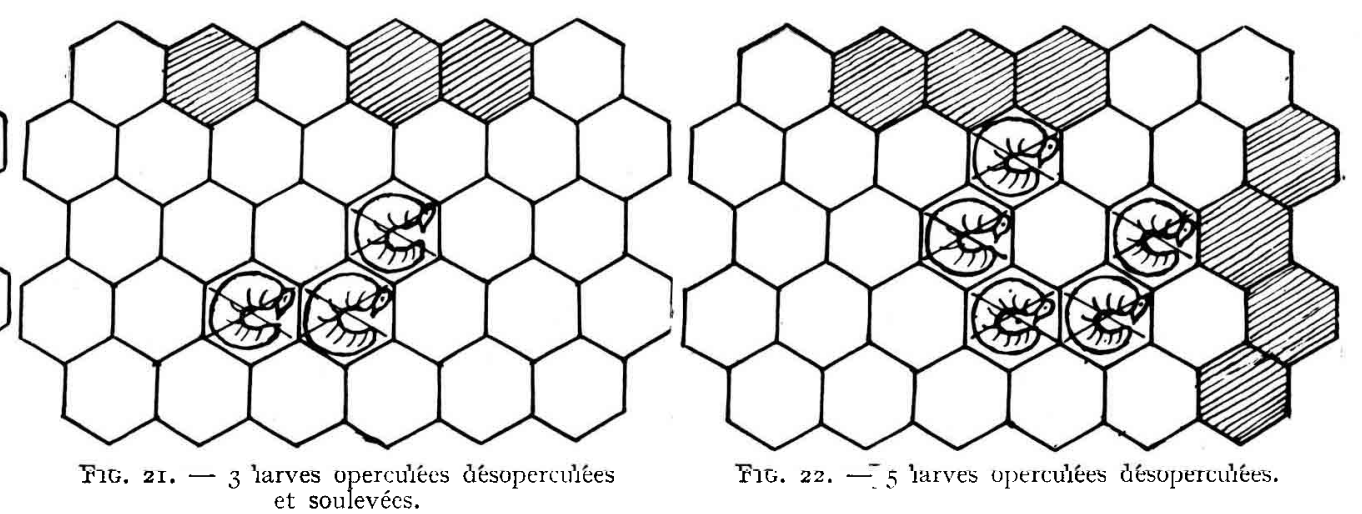



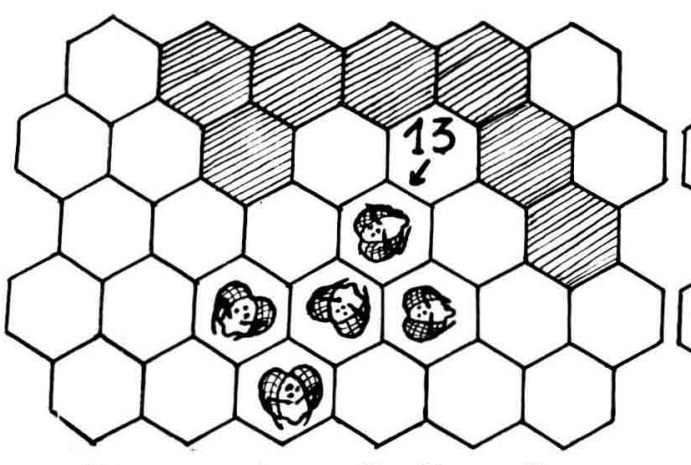

Fig. 23. -5 larves mâles désoperculées.
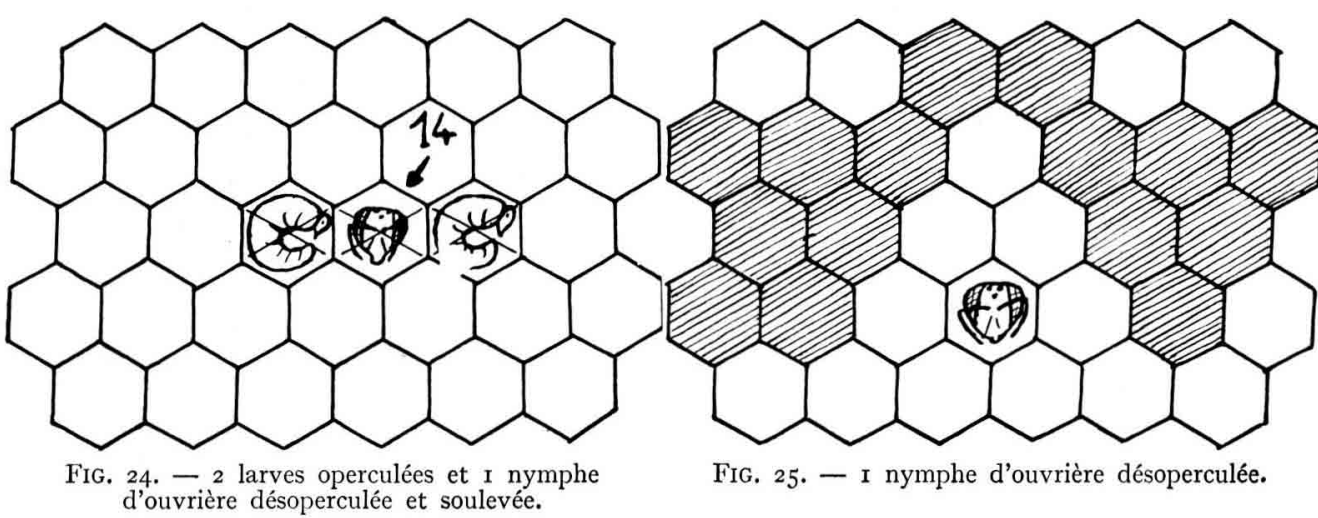
Pll

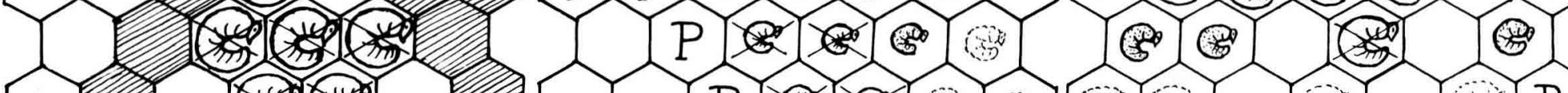

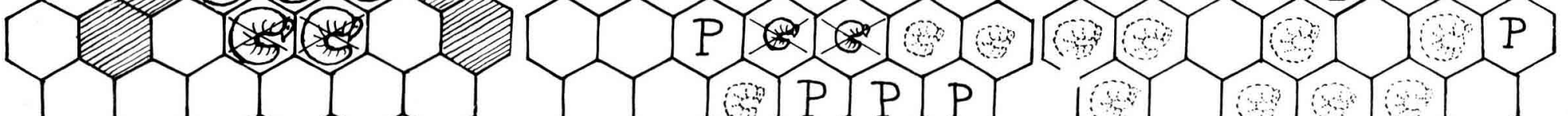
U

FIG. 27. - 4 petites larves transposées dans des cellules $\quad$ FIG. 28. -5 grosses larves transposées dans des cellules qui contenaient des petites larves. I larve transposée qui contenaient des grosses larves. 6 larves transposées
naturellement par les ouvrières.

(P)

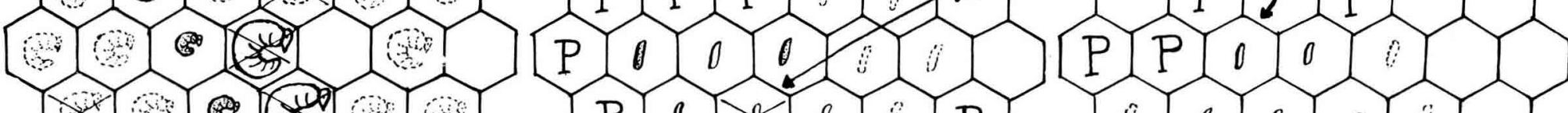
\& (2) Fig. 29. - 3 grosses larves transposées dans des cellules
qui contenaient des grosses larves. 4 larves transposées Fig. 30. -4 œufs soulevés. Fig. 3r. -7 œufs transposés.

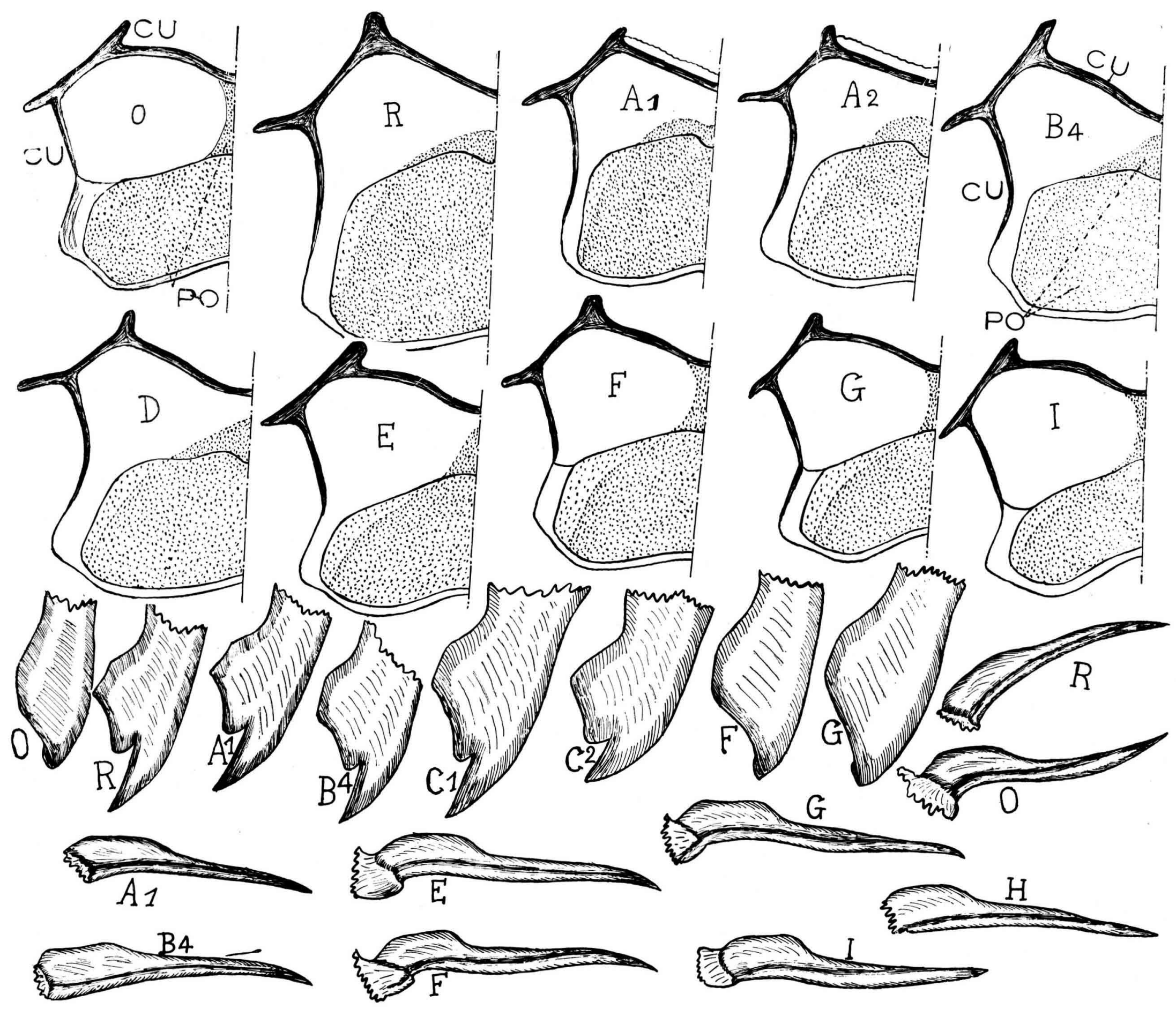

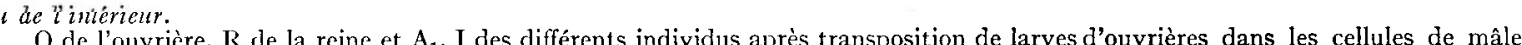
Cu : bord articulaire. I'o : Insertion des poils. Mandibules : $\mathrm{O}$ de l'ouvrière, $\mathrm{R}$ de la reine $\mathrm{A}_{1} . \mathrm{G}$ des autres individus. Aiguillons $: 0$ de l'ouvrière, $\mathrm{R}$ de la reine, $\mathrm{A}_{1}$. I des autres individus. Couvain operculé (cellule indiquée par la flèche suivant le nombre $\mathrm{I}$ sur la figure $\mathrm{n}^{\circ}{ }^{2}$ ). 



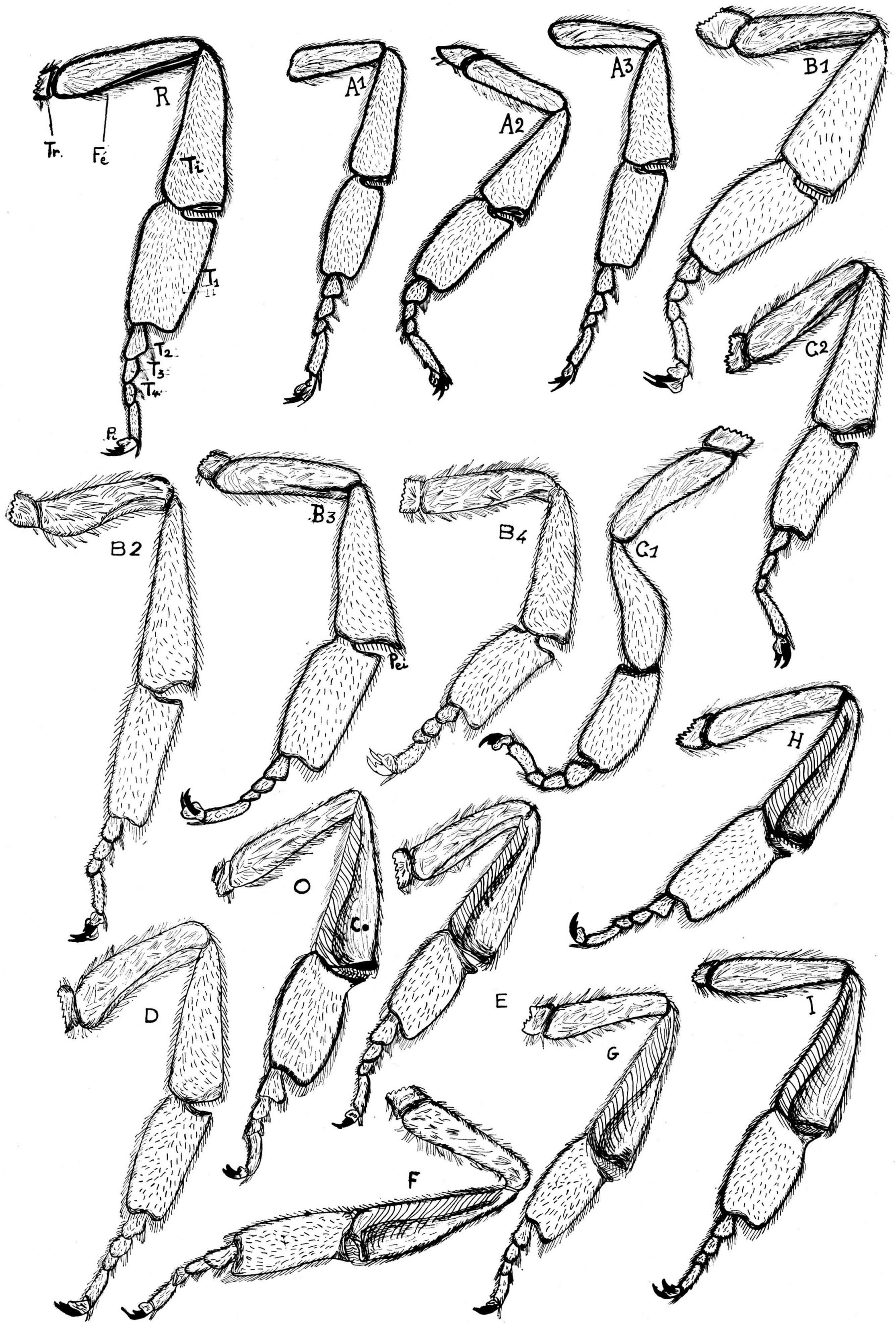

R. Patte postérieure de reine. O. Patte postérieure d'ouvrière.

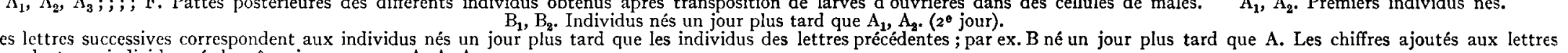

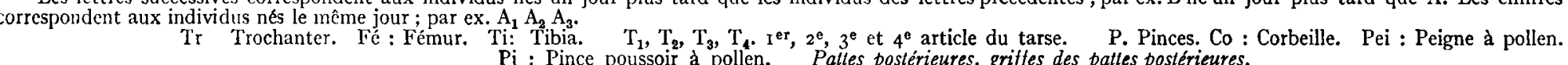




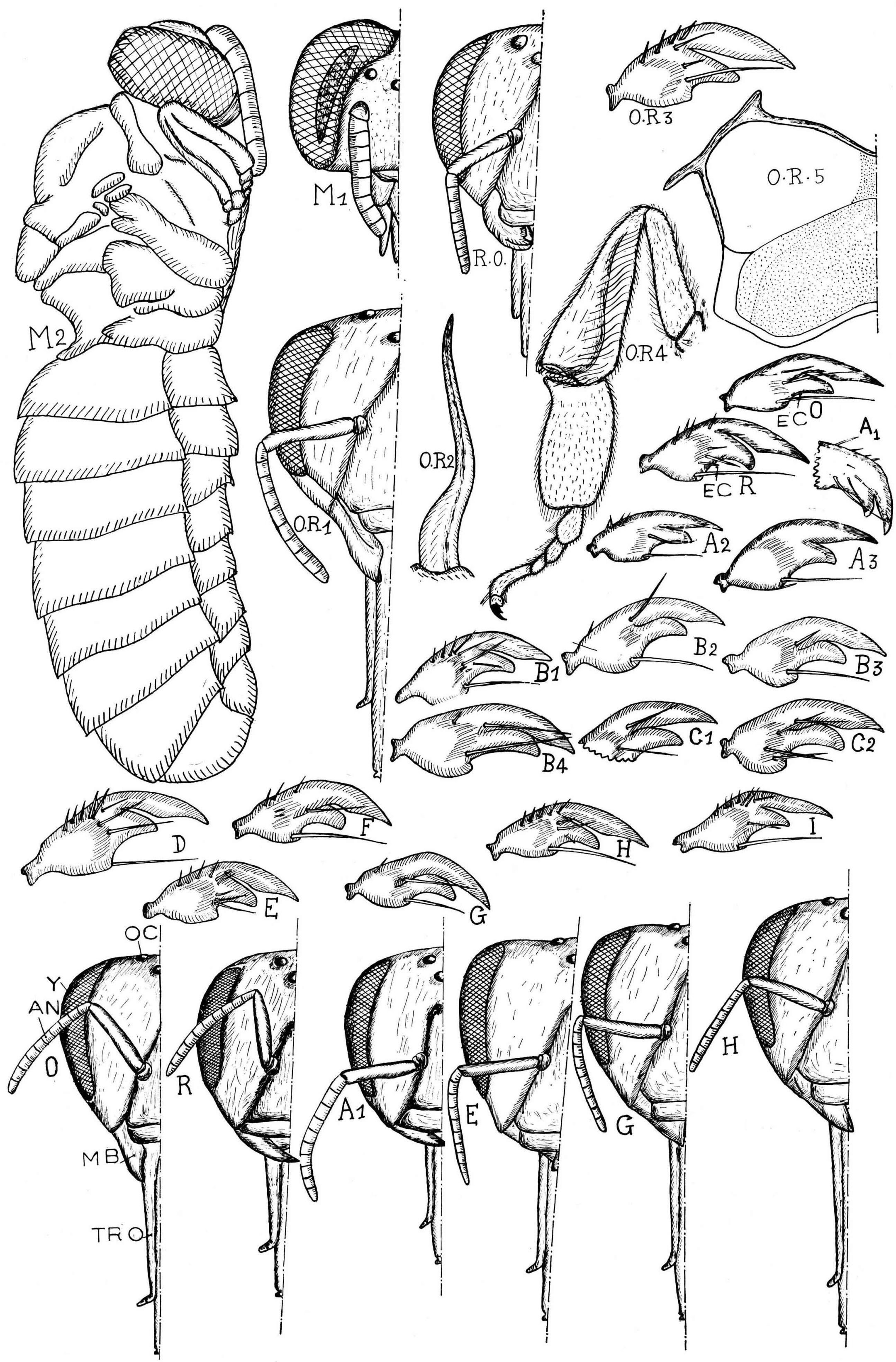

Moitiés de tétes des différents individus obtenus après transposition de larves d'ouvrières dans des cellules de mâles. R. Griffe de patte postérieure et demi-tête de reine. $M_{1}$ Moitié de la tête te ce. $Y:$ : Eil composé. $M_{3}$ Nymphe obtenue après transposition de larve de mâles dans les cellules d'ouvrières. 